This item was submitted to Loughborough's Research Repository by the author.

Items in Figshare are protected by copyright, with all rights reserved, unless otherwise indicated.

\title{
Real-time integration of prototypes in the product development process
}

PLEASE CITE THE PUBLISHED VERSION

http://dx.doi.org/10.1108/01445151311294621

\section{PUBLISHER}

(c) Emerald Group Publishing Limited

\section{VERSION}

AM (Accepted Manuscript)

\section{LICENCE}

CC BY-NC-ND 4.0

\section{REPOSITORY RECORD}

Liu, Bingjian, R.I. Campbell, and Eujin Pei. 2019. "Real-time Integration of Prototypes in the Product Development Process". figshare. https://hdl.handle.net/2134/12113. 
This item was submitted to Loughborough's Institutional Repository (https://dspace.lboro.ac.uk/) by the author and is made available under the following Creative Commons Licence conditions.

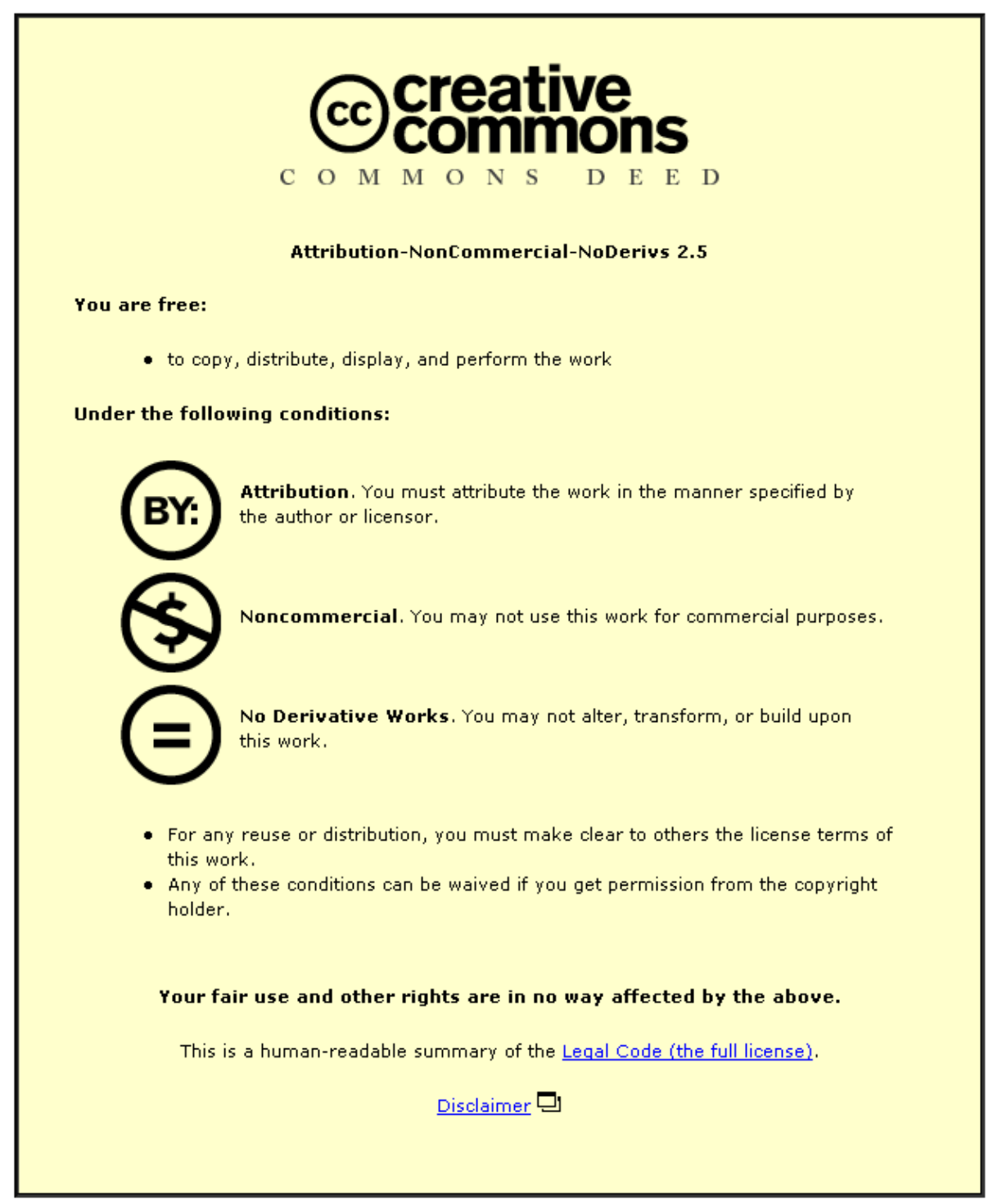

For the full text of this licence, please go to: http://creativecommons.org/licenses/by-nc-nd/2.5/ 


\section{Real-time Integration of Prototypes in the Product Development Process}

Authors:

B. Liu, Loughborough Design School, Loughborough University, UK

R.I. Campbell, Loughborough Design School, Loughborough University, UK

E. Pei, Department of Product \& Furniture Design, De Montfort University, UK

Purpose:

This paper identifies the strengths and weaknesses associated with physical and virtual prototyping and proposes an approach that utilises a real-time integration of both methods through an automated process.

Design / Methodology / Approach:

Following a literature review, the paper presents the results of a survey investigating the current use of prototyping. It then discusses a series of trials that were developed for the proposed tool.

Findings:

Physical and virtual prototypes are not competitive but rather complementary. An integrated real-time system would reduce cost and shorten the product design process.

Originality / Value:

This paper provides recommendations on how real-time integration of both physical and virtual prototypes could potentially streamline the new product development process.

Keywords: Real-time, integration, prototypes, product design 


\section{Introduction}

Prototyping is the pivotal activity that structures innovation, collaboration, and creativity in design (Hartmann \& Klemmer 2006). Repeated, efficient, and extensive use of prototypes can make a difference in the successful entry of new products into a competitive global market (Zorriassatine et al. 2003). In broad terms, prototypes can be classed into physical or virtual forms. They are created to finalise aesthetics and ergonomics, as well as to test, evaluate and validate the functional and technical aspects of the design (Pei et al, 2010). In a conventional approach, physical prototyping plays a very important role in New Product Development (NPD). In particular, it supports a concurrent, time-oriented approach and supports collaboration in teams composed of people from different functions and backgrounds (Vandevelde et al. 2002). In contrast, virtual prototyping is a relatively recent concept in design; and the term first being used in relation to virtual reality (Dai \& Göbel, 1994). The technology is a natural progression and an extension of Computer Aided Design techniques such as Finite Element Analysis. Due to its strength in reducing cycle time and cost, virtual prototyping has effectively replaced physical prototyping in several areas and will be used even more widely in the future (Huang \& Chen 1999). Despite this, Grimm (2005) argues that physical and virtual prototyping should not be seen as competitive but rather complementary technologies.

By understanding how physical and virtual prototyping technologies are used and how they interact with each other, there is a potential to develop tools that enable designers, engineers, manufacturers or other stakeholders in NPD to apply them concurrently in design, development and validation, thus achieving its maximum potential. The main challenge is to develop a prototyping method that can deliver instantaneous changes in both physical and virtual environments. In light of this, the aim of this research is develop a tool that can achieve real-time integration both prototypes through automation. The paper begins by reviewing the benefits and pitfalls of physical and virtual prototyping. It goes on to describe related work, following which results of a questionnaire survey and pilot trial are presented to specify what the tool should incorporate. Finally, the paper draws some conclusions and presents future research directions.

\section{Physical and Virtual Prototypes}

In the context of NPD, physical prototypes provide both tactile and visual evaluation of aspects including form, feel, surface finish, and so on. As most products incorporate some form of human interaction, this tactile advantage should be seen as highly desirable, particularly when interacting with customers (Campbell et al 2007) . In contrast, virtual prototyping is most often used when physical prototyping application are impractical, impossible or inefficient (Grimm 2005). Virtual prototyping allows the product to be replicated and tested in a digital environment and often within a CAD system to produce results. Examples of this include the testing of tensile strength, fluid dynamics, kinematic analysis and even visual evaluation. Virtual 
prototype has the key advantage of being faster (once the virtual prototype has been created), more cost effective and easier for modifying both prototype and test parameters. Hence, it becomes desirable to adopt both forms of technologies within NPD, since the strengths and advantages of one technology potentially addresses the weakness and limitations of the other. Gibson et. al (2005) proposed that physical and virtual prototyping are valuable techniques that can join together to form a more powerful tool for rapid development of complex products. This leads to a need for some form of integration between physical and virtual prototyping. As stated by Jain (2005), the integration of physical and virtual prototypes should yield shorter development cycles, fewer late-stage errors, and a higher return of investment.

For this study, "real-time integration of physical and virtual prototypes" shall refer to the synchronised changes that occur in both physical and virtual forms of prototyping. It is hoped that through a real-time integration, the benefits of these two forms of prototyping activities can be best used when needed so as to reduce time and cost.

\section{Related Work}

Previous research concerning the integration of physical and virtual models include those by Bruns (1998) who proposed the concept of a Graspable User Interface that could combine physical objects in a virtual environment. The system requires the use of a "data glove" when picking and moving physical objects. The movements are then tracked and replicated within a virtual environment containing virtual copies of the real-world object. This system allows users to transfer changes that take place in the physical world into a virtual environment, for instance making it possible for a circuit designer to build circuits in reality and in a virtual environment at the same time (Figure 1). However, the drawback is that this system was not able to alter the shape of the objects and could only assemble pre-defined objects. Therefore, the application of the system would be limited to later stages of NPD.

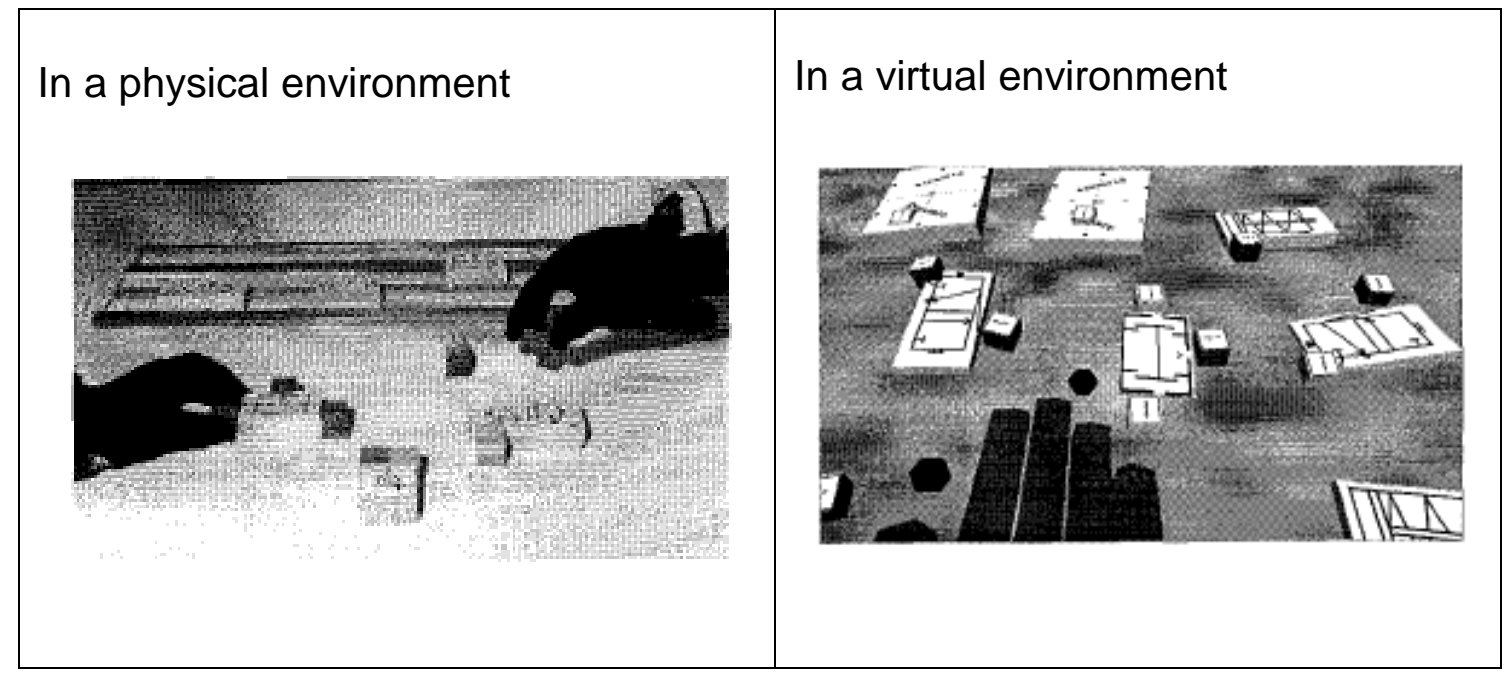

Figure 1. Building circuits simultaneously in both environments (Bruns 1998) 
Campbell (2003) further proposed an approach that would enable the simultaneous creation and modification of two analogous prototypes - one virtual and one physical. This would be achieved through real-time sharing of data between two different prototyping media, as illustrated in Figure 2. The approach was aimed at integrating both virtual and physical prototypes to facilitate greater user involvement in NPD. In the framework, both forms of prototypes are able to seamlessly cross over, replicate and undertake modifications when required.

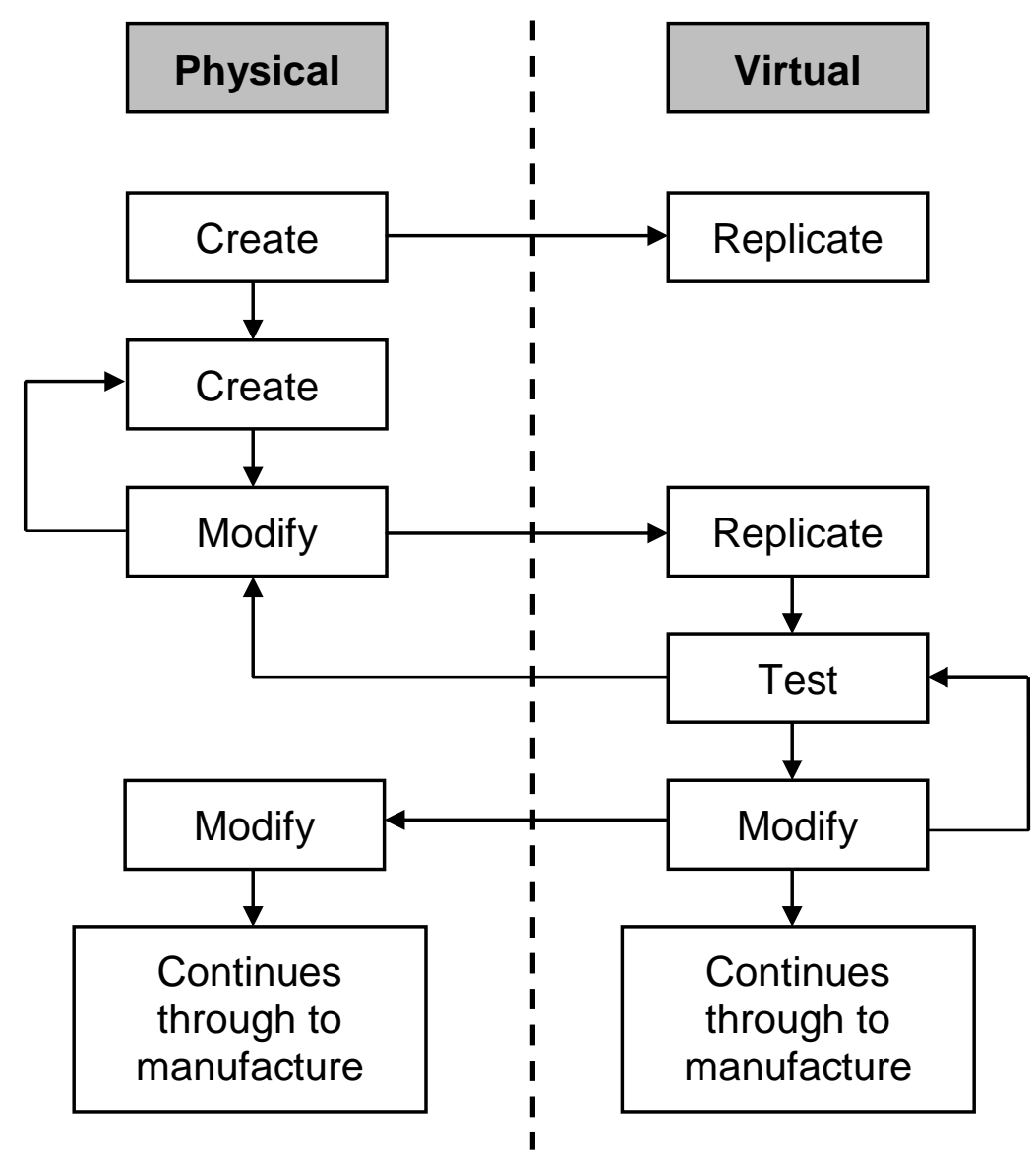

Figure 2. Integrating physical and virtual prototypes (Campbell 2003)

Parallel to Campbell's approach, Anderl et. al (2006) developed a tool known as parametric prototyping to achieve integration for equation-driven (parametric) and physical shape representation so as to optimise styling and design. They defined the parametric prototyping as a set-up of physical mockups and virtual models that are linked by an interface. The system comprises of a physical mock-up that is divided into several sections and each part is linked with a corresponding component in the digital space that is connected 
through a computer interface. Changes to the virtual part can be relayed to the physical component by means of electrical, mechanical and control mechanisms. Similarly, changes to the physical part can be transmitted to the corresponding part in the virtual space. Compared to other integration technologies, the most important advantage of this system is its achievement of bi-directional conversion. However, the purpose of this system is still limited; restricted to only the outer-styling of components and the changes were limited to the motion of the parametric parts in horizontal and vertical directions. In addition, the virtual model is incomplete and does not simulate the material properties, colour, texture and environment that are essential in NPD. As for the physical prototype, it cannot be used with product users to test aspects such as ergonomics and usability. Despite all of its limitations, the development of this tool meets the requirements of real-time integration between physical and virtual prototypes, such as data transfer between a virtual system and a physical component, and the bidirectional conversion of shape and size changes. In light of this, the next section discusses the questionnaire survey to elicit feedback from industry on the use of physical and virtual prototypes.

\section{Questionnaire Survey and Initial User Trials}

To better understand the use of physical and virtual prototypes in NPD, the authors undertook a questionnaire survey involving sixteen design consultancies and companies in the UK. The findings showed that around half of the respondents claimed they required the need for real-time integration of these two forms of prototyping. However, the practice of simultaneous use of physical and virtual prototype was seen as being in its infancy without any suitable or effective methods to integrate both forms in real-time.

Taking a step further, a simple trial was set up involving eight participants from a product design background to evaluate how the integration of physical and virtual prototypes could help them in NPD. The participants were designers and engineers working in companies, consultancies or academic institutions. For this trial, the participant was asked to test the backrest angle of a chair. When changes were manually made to the backrest angle, the CAD model of the chair in a digital space also changed (Figure 3).

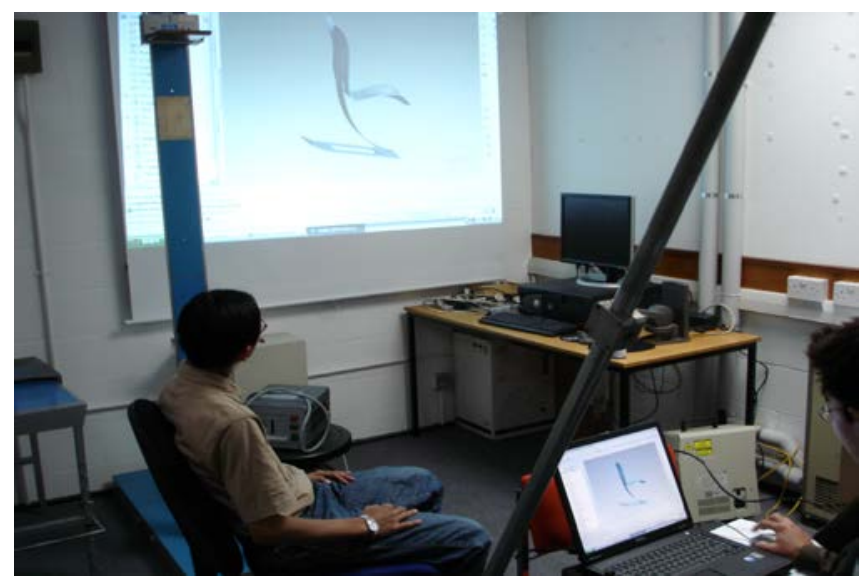


From the experiment, the user was able to see how the profile of the chair had been altered when the angle of the backrest was changed (Figure 4). The results showed that the simultaneous representation of changes to physical and virtual prototype was more helpful for evaluating their design. More importantly, because both models could be adjusted, no further prototypes were required to be built, thus saving cost and time to some extent. The outcomes of the two preliminary investigations showed that there was both potential need with benefits of having an integrated approach to physical and virtual prototyping for NPD.
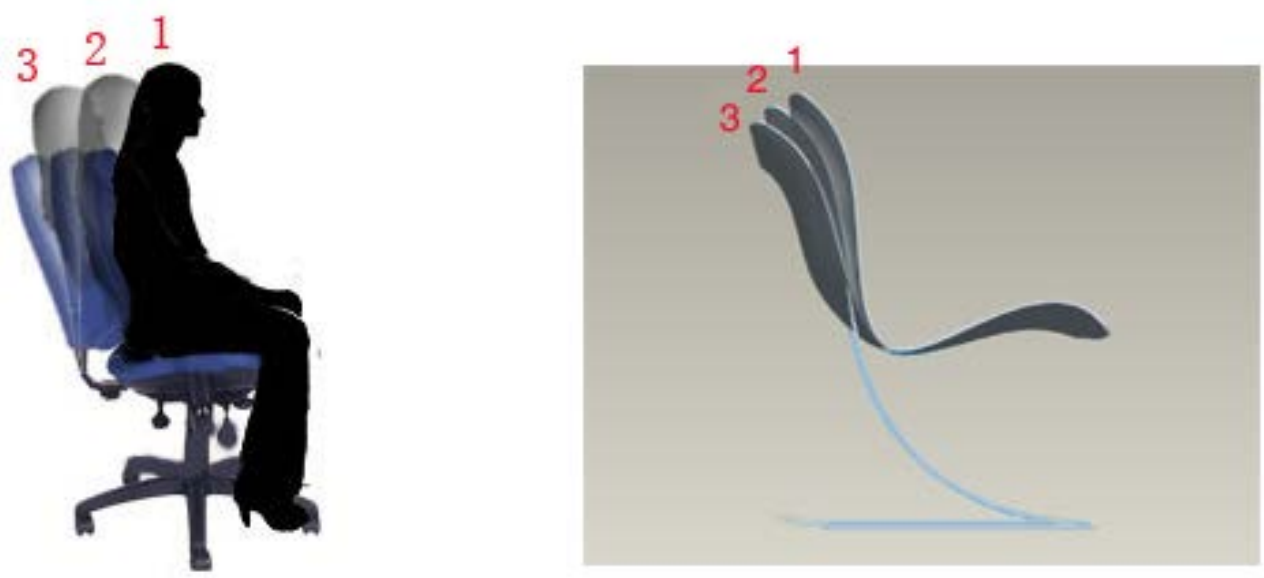

Figure 4. Corresponding changes of the physical and virtual chair

\section{Automated Prototypes and Trials}

Based on the theoretical knowledge gained from literature and findings from the survey and pilot study, it was decided to develop a more comprehensive system that would better integrate both prototype methods. As the infrastructure for the chair prototype was already in place, a decision was made to improve the structure of the test rig which would now comprise aspects of its height, as well as the angle of backrest. The height of the chair and angle of the backrest could be both adjusted by the user pressing relevant switches connected to two independent electric motors. A virtual model, was rebuilt using the Rhino CAD package that would simulate the aesthetical aspects of the chair. The digital model was linked to the physical interface by means of using the 'control points' feature embodied in Rhino.

In order to build the 'integrated link' between both prototypes, a standard computer mouse was used. The two sensors of the computer mouse were connected to the physical model whereby the horizontal sensor tracked the movement of the backrest in the horizontal direction, while the vertical sensor would track physical changes to the height of the seat. The movement of the physical model was transferred to the computer via the USB interface of the mouse; and its electrical signal was also used to move 'control points' in the virtual CAD model (Figure 5). Taking several measurements and comparing 
with the virtual model, the dimensional accuracy was determined as being within a 15\% margin of error. Figure 6 shows how the experiment took place. The participant was asked to sit on the chair and had the option of adjusting the height and the angle of backrest using the two switches. The designer was positioned in front of the computer and could instruct the user during the experiment. At the same time, the virtual CAD model was projected onto the screen to give the user a full view of the virtual model, allowing the participant to see changes simultaneously.

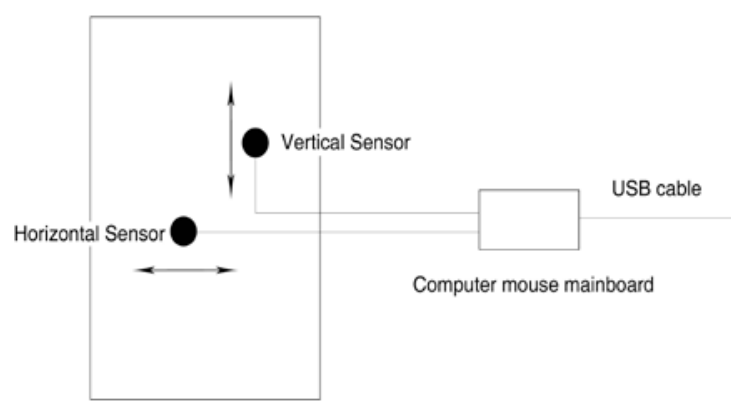

Physical Model

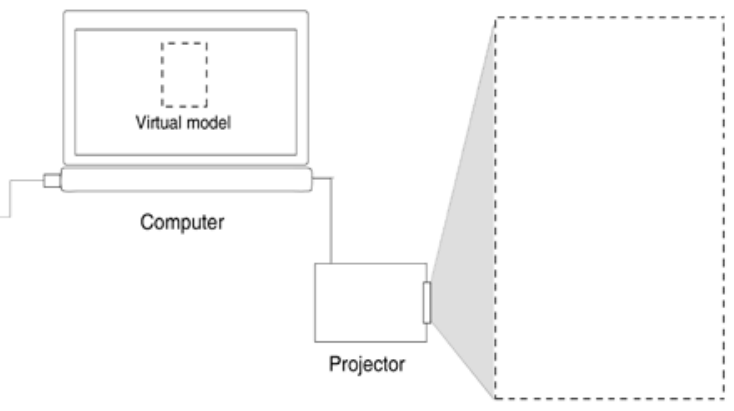

Virtual Model

Figure 5. Technical overview of the system

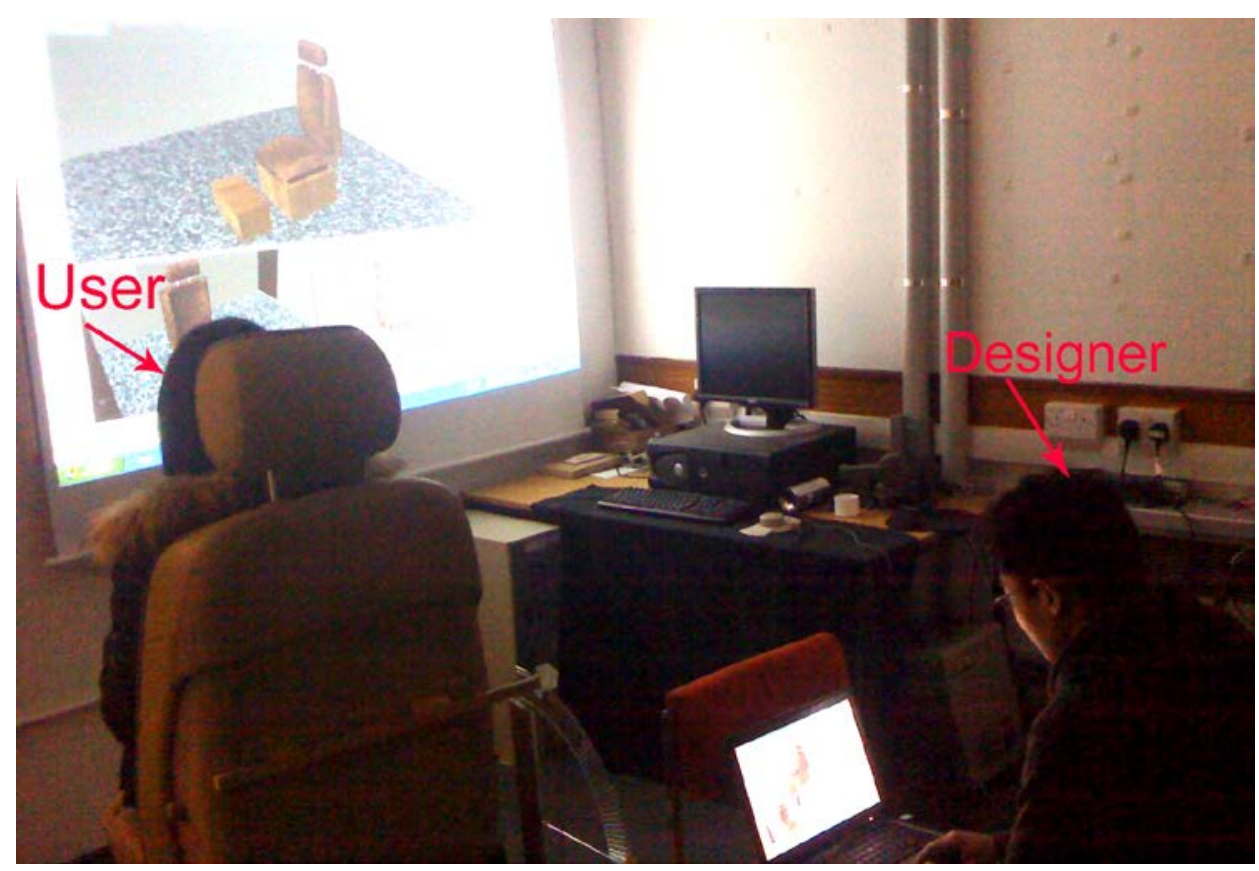

Figure 6: Set up of experiment

In total, eight pairs of participants (a user and designer) were involved in the trial. To fully compare the proposed method with a traditional product testing approach (where the physical and virtual models are tested separately), the whole experiment was divided into two stages. In the "disconnected" stage, 
the participants were asked to undergo the trial when the physical and CAD models were disconnected. At this stage, the user was asked to adjust the chair height and seatback angle to comfortable positions. After this, the designer measured the changes with a ruler and goniometer and had to manually input the data into the CAD model to update it. On average, the "disconnected" process took about seven minutes to complete. Next, for the "connected" stage, the user was asked to follow the same process, with the exception that now the CAD model would be automatically updated while the real chair was being adjusted. This process took about three and half minutes, on average, to complete. For this experiment, the key limitation was that the physical prototype was not able to be adjusted from the CAD model.

All participants were asked to complete a questionnaire to elicit feedback from the trials. The aim was to evaluate their satisfaction with the system. Two sets of questionnaires were developed and the questions for users and designers were different. The users' set of questions were concerned more about the system's usability and whether it would allow them to understand the design better. In contrast, the designers' set centred more on the efficiency of the tool as compared to having two distinct methods of prototyping. The findings showed that the majority of both sets of respondents felt that the system was easy to operate and that they were generally satisfied with the process of the trial. However, some of them commented that the physical prototype could be better built for greater accuracy and reliability. Other participants commented that the system would be most useful for designers, but were also concerned about the amount of time and effort required to build both prototypes. Most importantly, the respondents commented that the system was an excellent and novel way to simultaneously test the properties of both physical and virtual prototypes, allowing instantaneous changes to be seen. The secondary purpose of this trial was also to identify potential problems within the system and testing showed that most problems were technical. For instance, the structure of the physical mock-up was not robust enough and resulted in lack of accuracy.

\section{Specifications for Building Integrated Physical and Virtual Prototypes}

In order for real-time integration of physical and virtual prototypes to be fully realised, it became important to develop a framework that would define this concept. The framework should meet the following basic requirements:

i) It should be suitable for products with a range of sizes and complexity whilst recognising that not all products are suited to the approach.

ii) It should transfer all possible changes of the physical prototype to the virtual prototype, including the variation of linear dimensions and the modification of free form shape.

iii) The level of automation in physical prototyping should be improved to further reduce the time. 
There are some technologies that could be employed to meet these requirements. Motion capture technology has the potential to overcome the multi-dimensional problem. Widely used in the film industry, this technology can transfer movements of actors in the stage set to the computer. This is done by placing "markers" at key locations being worn by the actor, usually at movable joints and then using this to deduce the movement of the person. For the small sized product, a "data glove" could be a key enabling device for such an application. Wearing this glove and holding the prototype, the user could move their fingers in relation to the prototype, which could then be transferred to the virtual model instantaneously. This novel method offers the capability to test hand-held devices, as well as larger products such as furniture. In addition, the use of automation can improve the overall system.

When the related technologies are successfully embodied and the integration approach is further developed to meet all of the above requirements, it could become a generic decision support tool for a range of different types of product evaluation. Generally speaking, applying this integration approach will always involve the following steps:

1. Select aspects of the product need to be evaluated

2. Decide which are to be evaluated physically or virtually

3. Build adjustable physical and virtual prototypes

4. Link the physical and virtual prototype with appropriate sensors

5. Calibrate the physical and virtual prototypes so that they move in line

6. Ensure that both are presented in a similar scale / aspect ratio

7. Follow a detailed testing protocol

8. Obtain feedback

\subsection{Case Study - Design of a Motorbike}

As a form of transport, the motorcycle follows strongly the concerns of style as well as ergonomics. Here, it is chosen as an example to indicate the validity of the wider potential application of the generic prototyping integration tool. Each of the eight steps for implementation is described in the sections below.

\subsubsection{Product Aspects to be Evaluated}

Motorcycle manufactures aim to develop motorcycles with distinctive looks, sound, ride and performance. This indicates that the style, ergonomics, as well as engineering issues, are critical concerns for motorcycle design and manufacturing. All of these elements should be evaluated in the development of the motorcycle.

\subsubsection{Select Aspects to be Evaluated Physically or Virtually}

Physical prototypes usually have the advantage in ergonomic issues, while virtual prototypes have more benefits in testing aesthetics and predicting performance. In this case, the foot rest position, seat height and handle bar position, which attract more attention from an ergonomics perspective, could be evaluated physically. While the styling and the paint colour, could be evaluated virtually. Performance aspects such as aerodynamics and acceleration could also be evaluated using virtual prototypes. 


\subsubsection{Building the Physical and Virtual Prototypes}

The physical prototype should be built from standard parts and the elements to be evaluated, should be adjustable. The virtual prototype should be built with mainly aesthetic factors in mind, such as texture and the paint colour.

\subsubsection{Linking the Prototypes with Appropriate Sensor Technologies}

When the physical and virtual prototypes have been constructed and modelled, the next step is to link them with appropriate sensor technologies. For the seat height, handle bar and foot rest position, the movements would generally be linear and sensors similar to those from a mechanical computer mouse could be used. For the seat cushion which will have shape deformation, haptic technology could be considered as a possible solution.

\subsubsection{Calibrating the Physical and Virtual Prototypes}

It is likely that each element of the prototype would need to be tested and calibrated separately. Finally, the whole system can then be tested to verify if there are any issues in conflict.

\subsubsection{Selecting the Method of Presentation}

The testing of prototypes could take place in a controlled area whereby the physical prototype could be located in front of screen. This set up would be similar to the simulators being developed by the Human-Motorcycle Interaction research unit at the University of Nottingham (Stedmon 2010) where it could also show the product in a realistic (driving) environment.

\subsubsection{Finalising the Test Protocol}

When the system has been correctly calibrated and a testing environment put in place, the validation can start by following a protocol. It should contain a list of components that will be tested; and how to operate the prototypes. At the same time, the documentation should also provide recommendations on how to operate the recording devices. A questionnaire or interview for feedback should also be designed at this stage.

\subsubsection{Obtaining Feedback from the Participants}

After completing the evaluation, feedback should been collected by either a questionnaire or a face-to-face interview, or if possible, by both means. The outcome of the feedback will then be used for the next development of the motorcycle design.

\section{Conclusions and future work}

In conclusion, real-time integration of physical and virtual prototypes has shown to be a novel way of improving the evaluation of products within the 
context of NPD. This system considers three key aspects: firstly, it makes the best use of the advantages of both physical and virtual prototypes; secondly, it is compatible with most 3D modelling software that is commonly used in NPD; thirdly, it should improve the evaluation process, thereby reducing time and the need for additional prototypes. The user trials with an integrated virtual/physical prototype of a chair showed that both consumers and designers found the method to be advantageous. The consumers felt that they could understand the product better and have greater involvement in modifying the design. At the same time, the designers felt that they were able to change the design seamlessly according to customer feedback without the need for a new prototypes to be constructed, reducing time and cost. However, some criticisms include the reliability of the physical prototype; and the need to fully demonstrate an integrated system for real-time industrial design development.

A more generic approach to implementing the method has been presented and illustrated using the example of motorcycle design. A key consideration is selecting suitable sensor devices to connect the physical and virtual prototypes. While the mechanical computer mouse was able connect both prototypes and achieve a simultaneous update, the drawback was in its accuracy, with more precise measurement being required. A promising alternative would be to use motion capture devices.

Finally, the proposed method only demonstrates a unidirectional integration of physical and virtual prototypes, i.e. changes in the physical prototype leading to simultaneous changes in the virtual prototype. It is thus envisioned that the next stage of this research is to achieve a bi-directional integration of both physical and virtual prototypes that could potentially involve greater use of sensors and robotics. 


\section{References}

Anderl, R., Klug, L., and Mecke, K. (2006). "Advanced prototyping with parametric prototypes." Digital Enterprise Technology (DET). Setúbal, Portugal. September 18-20., available from http://Itodi.est.ips.pt/det2006/papers/Physical/f102_PH4.pdf Accessed on 7 May 2009.

Bruns, F. W. (1998). "Integrated real and virtual prototyping" IECON proceedings, Industrial Electronic Conference, Vol. 4, 1998, pp. 2137-2142.

Campbell, R. I. (2003). "Real time integration of virtual and physical prototyping." International conference on advanced research in virtual and rapid prototyping. Leiria, Portugal. October 1-4, 2003. pp.31-37

Campbell, R.I., de Beer, D.J., Barnard, L.J., Booysen, G.J., Truscott, M., Cain, R., Burton, M.J., Gyi, D.E. and Hague, R.J.M., (2007) "Design Evolution through Customer Interaction with Functional Prototypes", Journal of Engineering Design, 18(6), 2007, pp 617-635,

Dai, F. and Göbel, M. (1994), "Virtual Prototyping - An Approach Using VRtechnique," Proceedings of the 1994 ASME Computers in Engineering Conference, Minneapolis, MN, 1994.

Gibson, I., Gao, Z. and Campbell, R. (2005). "A comparative study of virtual prototyping and physical prototyping." International journal of manufacturing technology and management. Vol. 4 (No.6). pp. 503-522

Grimm, T. A. (2005). "Virtual Versus Physical: Will Computer-Generated Virtual Prototypes Obsolete Rapid Prototyping." Time-Compression Technologies, Vol. 13 (No.2).

Hartmann, B. and Klemmer, S. R. (2006). "Reflective Physical Prototyping through Integrated Design, Test, and Analysis." The nineteenth annual ACM Symposium on User Interface Software and Technology. Montreux, Switzerland. October 15-18 2006.

Huang, C. D. and Chen, Z. J. (1999). "Virtual prototyping and virtual prototype in Control System." Journal of Beijing University of aeronautics and astronautics Vol. 25(No.3).

Jain, S. (2005). "Integrating Simulation and Physical Testing Leads to Shorter Design Cycles." Time-compression technologies. Vol. 13(No. 3)

Liu, B. J. and Campbell, R. I. (2008). "Real time integration of user preferences into virtual prototypes" Proceedings of DSR 2008, Design Research Society Biennial Conference, Sheffield, UK, 16-17 July 2008. 
Pei, E., Campbell, R.I. and Evans, M.A., "Development of a tool for building shared representations among industrial designers and engineering design", CoDesign Journal, 6(3), 2010, p139-166, ISSN 1571-0882.

Stedmon A. W. (2010). Retrieved on 15 Aug 2011 from http://hcdi.brunel.ac.uk/seminardetails.aspx?sid=2

Vandevelde, A., Van Dierdonck, R. and Clarysse, B.. (2001). "The role of physical prototyping in the product development process," Vlerick Leuven Gent Management School Working Paper Series 2002-7, Vlerick Leuven Gent Management School.

Zorriassatine, F., Wykes C., Parkin R. and Gindy N. (2003). "A survey of virtual prototyping techniques for mechanical product development." Proceedings of the I MECH E Part B. Journal of Engineering Manufacture, Vol. 217(No.4), 1 April, pp. 513-530. 